\title{
Control of Active Nematics with Passive Liquid Crystals
}

Pau Guillamat, Jordi Ignés-Mullol*, and Francesc Sagués

Department of Materials Science and Physical Chemistry, and Institute of Nanoscience and Nanotechnology, University of Barcelona, Barcelona, Catalonia, Spain

* Marti i Franquès 1, 08028 Barcelona, Catalonia, Spain. E-mail: jignes@ub.edu

Author contact information:

\section{Pau Guillamat}

Email: pauguillamat@gmail.com

Phone: +34 934039252

FAX: +34 934212131

\section{Jordi Ignés-Mullol}

Email: jignes@ub.edu

Phone: +34 934039290

FAX: +34 934212131

\section{Francesc Sagués}

Email: f.sagues@ub.edu

Phone: +34 934021242

FAX: +34 934212131 


\section{Control of Active Nematics with Passive Liquid Crystals}

Motor-proteins are responsible for transport inside cells. Harnessing their activity is key towards developing new nano-technologies, or functional biomaterials. Cytoskeleton-like networks result from the self-assembly of subcellular autonomous units. Taming this biological activity bottom-up may thus require molecular level alterations compromising protein integrity. Taking a top-down perspective, here we prove that the seemingly chaotic flows of a tubulin-kinesin active gel can be forced to adopt well-defined spatial directions by tuning the anisotropic viscosity of a contacting Smectic-A liquid crystal. Different configurations of the active material are realized, when the thermotropic liquid crystal is either unforced or commanded by a magnetic field. The inherent instability of the extensile active fluid is thus spatially regularized, leading to organized flow patterns, endowed with characteristic length and time scales. Our finding paves the way for designing hybrid active/passive systems where ATPdriven dynamics can be externally conditioned.

Keywords: active matter; active nematic; focal conics, Smectic-A; magnetic-field alignment;

\section{Introduction}

Cytoskeleton reconstitutions are structured soft systems displaying characteristics of selfassembled colloidal dispersions, but, distinctively, they are active ${ }^{1-11}$. Under suitably constrained conditions, and with a steady supply of ATP, these new types of materials feature emergent modes of self-organization that arise from the collective behavior of individual biomolecules. Besides posing fascinating questions, related to the permanent and intrinsic non-equilibrium nature of these systems ${ }^{12,13}$, the fact that they constitute in vitro models for the intra-cellular milieu suggests a potential for the development of new responsive biomaterials. For instance, one could envision to externally commanding the preferred flow lines, deformation directions, or intrinsic entanglements of these active gels, although a successful strategy in this direction has yet to be demonstrated. The complexity and specificity of the involved components seems to preclude an a priori 
process of protein engineering. It seems thus advisable to devise a control strategy with the potential of being exerted on any viable active gel. Actuating by means of either an electric or a magnetic field is unlikely, given the high ionic strength of these preparations, which screens electric responses, and the low magnetic susceptibilities that make them insensitive to modest magnetic forces. On the other hand, one could resort to the confinement of the active material in patterned microfluidic channels, which will determine the direction and time scales of its spontaneous active deformations. This procedure has the disadvantage of requiring complex biochemical functionalization of the inner surfaces to render the substrates biocompatible but, even if successful, this strategy will be neither versatile nor in situ reconfigurable. Here, we demonstrate a radically different approach by interfacing the aqueous active gel with a thermotropic liquid crystal that features the Nematic to Smectic-A (SmA) transition at a biocompatible temperature ${ }^{14}$. We take advantage of the of the fact that the Nematic phase can be easily aligned under a modest magnetic field, and of the fact that the SmA phase features a dramatic anisotropy in the shear viscosity. We employ these features to tame the seemingly chaotic flows of protein gels.

\section{Experimental Section}

\section{Preparation of the Active Nematic}

As a precursor of the active nematic, we prepare an active gel of tubulin microtubules (MT) crosslinked by clusters of the molecular motor kinesin following the protocols detailed elsewhere ${ }^{15}$. Briefly, the MT are polymerized from bovine brain tubulin (a gift from Z. Dogic's group in Brandeis University) with a typical length $1-2 \mu \mathrm{m}$. For fluorescence microscopy, a fraction of the tubulin (3\%) has been fluorescently labelled with Alexa-647. The motor protein kinesin-1 from Drosophila Melanogaster is expressed 
in E. Coli, purified, and incubated in the presence of the protein streptavidin. This leads to the assembly of clusters that contain, on average, two of the biotilynated motor proteins. The MT are mixed with a solution containing the non-adsorbing polymer polyethilene glycol (PEG, 20kDa) and the kinesin clusters. PEG acts as a depleting agent, promoting the bundling of the MT into filaments, while the motor clusters act as crosslinkers of the gel. In the presence of Adenosine Triphosphate (ATP), kinesin promotes the sliding of MT bundles of opposite polarity, thus resulting in an extensile active gel. A PEG-based triblock copolymer surfactant (Pluronic F-127) is added to the protein mixture. This leads to the formation of a biocompatible interface when the liquid crystal and the aqueous medium are put into contact, and promotes the depletion of the active gel towards the soft interface, which leads to the formation of the quasi-two-dimensional active nematic when the filaments develop long-range orientational order.

\section{Experimental Setup}

The water/LC interface is prepared in a custom flow cell made using the commonly used transparent elastomer PDMS placed onto a glass plate coated with a hydrophilic and bio-inert polyacrylamide brush. The cylindrical cavity is filled with $50 \mu \mathrm{L}$ octylcyanobiphenyl (8CB, Nematel) and $1 \mu \mathrm{L}$ of the aqueous protein suspension is injected between the oil and the hydrophilic substrate, which is coated by the water phase. The system is heated to $35^{\circ} \mathrm{C}$ to promote the transition of $8 \mathrm{CB}$, which is in the $\mathrm{SmA}$ phase at room temperature, into the less viscous Nematic phase.

\section{Temperature Control and Magnetic Field Application}

Polarizing and fluorescence microscopy is carried out in a custom optomechanical setup built around a custom-made permanent magnet assembly that provides homogeneous planar magnetic field in a region much larger than the field of view, with a maximum 
strength of $0.4 \mathrm{~T}$. The magnet is a Halbach array of $25.4 \mathrm{~mm}$ commercial cubic magnets, custom designed and built as described elsewhere ${ }^{15}$. Samples are held inside a thermostatic oven build with Thorlabs SM1 tube components and tape heater, and controlled with a Thorlabs TC200 controller.

\section{Observation and Characterization}

Routine observation of the active nematic is performed by means of a conventional epifluorescence microscope, while laser-scanning confocal microscopy (both in fluorescence and reflection modes) is employed when sharper images are required.

\section{Results and discussion}

\section{Formation of rotating mills in contact with Toroidal Focal Conic Domains}

The chosen active material is based on the self-assembly of tubulin, from protein monomers all the way up to filamentary bundles of micron-sized stabilized microtubules ${ }^{6,16}$.The latter are cross-linked and sheared by clusters of ATP-fueled kinesin motors, which are directed towards the plus ends of the microtubules. Interfilament sliding thus occurs in bundles containing microtubules of opposite polarity (Fig. 1A). This mixture self-assembles into an extensile active gel ${ }^{17-19}$, continuously rebuilt following bundle reconstitution, and permanently permeated by streaming flows. An alternative preparation, more suited to our purposes, consists in depleting this bulk material towards a biocompatible soft and flat interface, where filaments continuously fold and adopt textures typical of a two-dimensional nematic ${ }^{6}$. This active layer appears punctuated by a steady number of continuously renovated microtubule-void regions that configure semi-integer defect areas. Although we focus on flat interfaces, the latter can be also curved, giving rise to interesting defect accommodation dynamics, and occasionally to intriguing deformation modes of the, in this way prepared, active 
vesicles $^{20}$. In our case, a layer of active gel is confined between a biocompatible hydrophilic rigid surface (bottom) and a (top) volume of the liquid crystal (LC) octylcyanobiphenyl (8CB), which features a Nematic/Smectic-A transition at around $33^{\circ} \mathrm{C}$, compatible with protein activity (Fig. 1B). The LC/water interface is stabilized with a polyethylene glycol (PEG)-based triblock copolymer surfactant.

To better appraise the role played by the contacting structured interface on the active material, the top passive liquid crystal is initially prepared in its Nematic phase. On average, the $8 \mathrm{CB}$ mesogen molecules lay parallel to the $\mathrm{LC} /$ water interface, due to the influence of the polymeric surfactant, and perpendicular to the LC/air interface far from the active layer. In the presence of isotropic oils, the contacting active nematic features the well-known disordered swirling motion, characterized by the formation and annihilation of defects ${ }^{21-24}$, accompanying the spontaneous folding of the bundled microtubules. Although nematic $8 \mathrm{CB}$ is anisotropic, shear viscosities along different directions are of the same order of magnitude, and do not trigger any observable differences when compared with the contact with isotropic oils (Fig. 2, A-C). It is known, in the latter case, that there is a strong hydrodynamic coupling between the active and the passive fluids. Indeed, the oil viscosity influences both the density and velocity of proliferating defects in the active nematic. This gives us a clear indication that the contact of the active and passive structured materials is robust, persistent, and stable enough to foresee stronger effects when the order of the passive phase is further upgraded to the more anisotropic Smectic-A phase.

A temperature quench below $33.4{ }^{\circ} \mathrm{C}$ triggers the reversible transition of $8 \mathrm{CB}$ into the lamellar Smectic-A phase. This has a dramatic impact on the interfacial rheology and on the dynamics of the active nematic. Layers in the Smectic-A phase are perpendicular to the $8 \mathrm{CB}$ molecules, which remain parallel to the LC/water interface. Free-energy 
minimization constraints, related to the boundary conditions at the interfaces, result in the formation of the so-called toroidal focal conics ${ }^{14}$. These are polydisperse circular domains that form a fractal tiling called Apollonius gasket ${ }^{25}$. Inside the domains, radially-oriented mesogen molecules arrange concentrically at the oil/water interface (Fig. 2D). Flow perpendicular to the Smectic layers is severely hindered and, contrarily, is highly facilitated along them. As a result, the local interfacial shear stress experienced by the active material is markedly anisotropic. Stretching of the bundled filaments thus occurs preferably along circular trajectories centered with the focal conics, which organize the active nematic in confined rotating mills (Fig. 2E). Flows can be traced following the motion of the $+1 / 2$ comet-like defects, and become fully apparent from time-averaged snapshots of the active pattern (Fig. 2F). Dark spots, where filament occupancy is lowest, appear then in register with the center of large focal conics in the Smectic-A oil. Increasing the temperature, the Smectic phase of the passive liquid crystal melts down and, as expected, the rotating mills are completely and immediately dismantled, recovering the typical textures of the active nematic. Filamentary bundles of the active material have an intrinsic maximum curvature that cannot be exceeded. As a consequence, only focal conics above a threshold diameter, which we have estimated at around $40 \mu \mathrm{m}$ in the experiment shown in Fig. 2, are able to coordinate the flow of the underlying active material. From a topological perspective, confined rotating filaments organize a topological singularity of charge $S=+1$, which corresponds to a full rotation around the domain center (Fig. 3). The semi-integer defects, which accompany rotating filaments, are permanently renovated, even their number may change to a small extend, assembling and disassembling continuously the core structure of the rotating mill. At all times, however, a balance is established such that the arithmetic sum of topological charges inside a single domain adds up to one 
(Fig. 3). This collective precession is accompanied by a periodic modulation of the velocity of the active flows.

\section{Reversible alignment of the active nematic with a magnetic field}

A tighter control of the active flow is achieved by directly actuating on the contacting thermotropic liquid crystal. To this purpose, we take advantage of its large (positive) diamagnetic anisotropy that enables to align a macroscopic volume of the material with uniform magnetic fields of the order of a few $\mathrm{kG}$, easily attainable with a permanent magnet array. By a slow temperature quench of $8 \mathrm{CB}$ from the nematic to the SmecticA phase in presence of a $4 \mathrm{kG}$ magnetic field parallel to the $\mathrm{LC} /$ water interface, we induce the formation of a layer of $8 \mathrm{CB}$ molecules uniformly aligned with the magnetic field. In this situation, the Smectic planes are oriented perpendicularly both to the interface and to the magnetic field (Fig. 4). It is well-known in the literature that this bookshelf geometry, robustly kept after removal of the magnetic field, results in a liquid that flows easily when sheared along the lamellar planes, but that responds as a solid to stresses exerted in the orthogonal direction ${ }^{14}$. Polarizing optical microscopy confirms the formation of this aligned smectic-A layer (Fig. 4A) that includes dislocations in the aligned lamellar planes, which propagate into the bulk of the material with the so-called parabolic focal conic domains. In contact with this interface, the turbulent active nematic experiences markedly anisotropic shear stresses, which result in a rapid rearrangement of the flow pattern that consists now in bands of uniform width, perpendicular to the magnetic field (Figs. 4 B-E). More precisely, stripes of densely stacked microtubules that appear bright in the fluorescence images, appear alternated with dark lanes of moving defects, along which antiparallel flow velocities are easily recognized, and clearly visualized using particle tracers. Negative defects are, in this situation, more difficult to localize, although the global topological requirement 
mentioned earlier applies here to secure a zero total charge. As before, this organized structure is immediately and totally reset, to recover the standard well-mixed configuration of the active material, when reversing the temperature quench back to the Nematic phase of the thermotropic mesogen. Moreover, by previous transiting back and forth through the passive nematic $8 \mathrm{CB}$ phase, the orientation of the active pattern can be readily and reversibly in plane-rotated by redirecting the magnetic field at will (Fig. 4 D, E). The striped pattern defines a characteristic wavelength, which depends on the activity of the sample. This characteristic spatial periodicity is associated to a typical length scale in the active material, $l_{\alpha}$, that has been predicted in theoretical studies to decrease with the square of the activity, and governs the decay of the flow vorticities and the director fields for unconfined active nematics ${ }^{26}$. By approximating the activity parameter as $\alpha \sim \ln [A T P]$, we recover the predicted scaling of $l_{\alpha}($ Fig. 5B).

Within stripes, bundles of microtubules are packed in parallel arrangements oblique to the lanes of moving defects (Fig. 6A, B). This microtubule disposition, although apparently motionless, is prone to suffer the bending instability intrinsic to any extensible active material ${ }^{27,28}$. Following such instability, pairs of complementary halfinteger defects are created and either they annihilate in pairs or incorporate into opposite preexisting lanes (Fig. 6C). Once defects escape from their birth areas, the striped regions are restored with minimal rearrangements. Such apparently vulnerable episodes of the active material extend coherently over regions of arbitrary extension but always commensurate with the stripe width, and occur periodically with a striking regularity. We conjecture that this kind of periodic "avalanches" arise from the intrinsic dynamics of the sheared microtubules, and provide a breakdown mechanism that the active 
material has at its disposition to repeatedly release the extensile tensional stress accumulated in the stripes of the active material.

In summary, our work demonstrates that the disordered flow patterns of an active twodimensional nematic can be controlled to follow preassigned directions. This is achieved by means of the anisotropic shear stress exerted at the interface by a thermotropic liquid crystal layer whose orientation can be externally commanded with a magnetic field. The ordered active material clearly reveals its characteristic time and length scales, which arise from non-equilibrium biochemical processes at the molecular level. The versatility, reversibility and robustness of such strategy should be considered as a proof of concept for the taming of active subcellular materials.

\section{Acknowledgments}

The authors are indebted to Z. Dogic and S. DeCamp (Brandeis University), and the Brandeis University MRSEC Biosynthesis facility for their assistance in the preparation of the active gel. We thank B. Hishamunda (Brandeis University), and M. Pons, A. LeRoux, and G. Iruela (Universitat de Barcelona) for their assistance in the expression of motor proteins. Funding has been provided by MINECO (project FIS 2013-41144P). P.G. acknowledges funding from Generalitat de Catalunya through a FI-DGR PhD Fellowship. Brandeis University MRSEC Biosynthesis facility is supported by NSF MRSEC DMR1420382. 


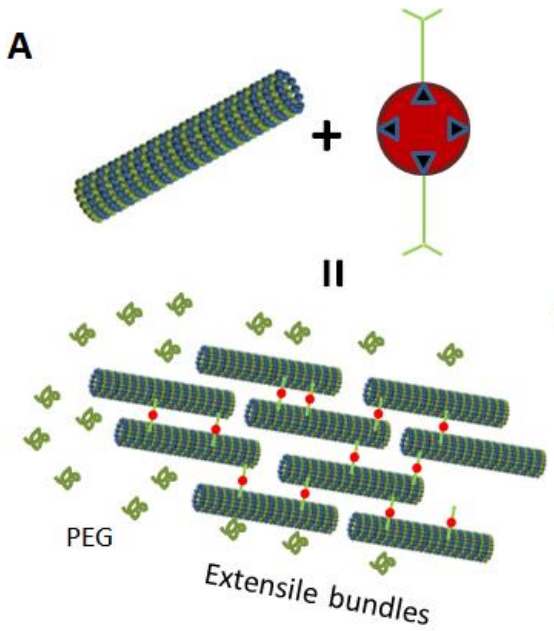

B

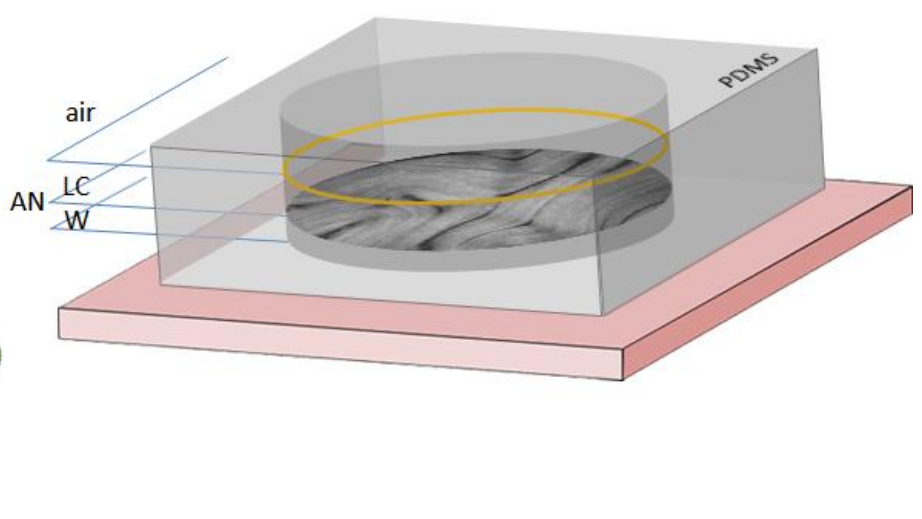

Figure 1. A: Tubulin microtubules are crosslinked into extensile bundles by clusters of kinesin motor proteins. Depletion interaction, originated by PEG present in the protein suspension, helps to condense the bundles. B: Flow cell used in the experiments. A $5 \mathrm{~mm}$ deep, $8 \mathrm{~mm}$ in diameter cylindrical cavity, custom made with the elastomer PDMS, is bound to a hydrophilic, biocompatible glass plate. The aqueous protein solution is in contact with the substrate, and a volume of LC is placed on top, defining the LC/water interface where the active nematic (AN) forms. The LC is open to the air at the top. 


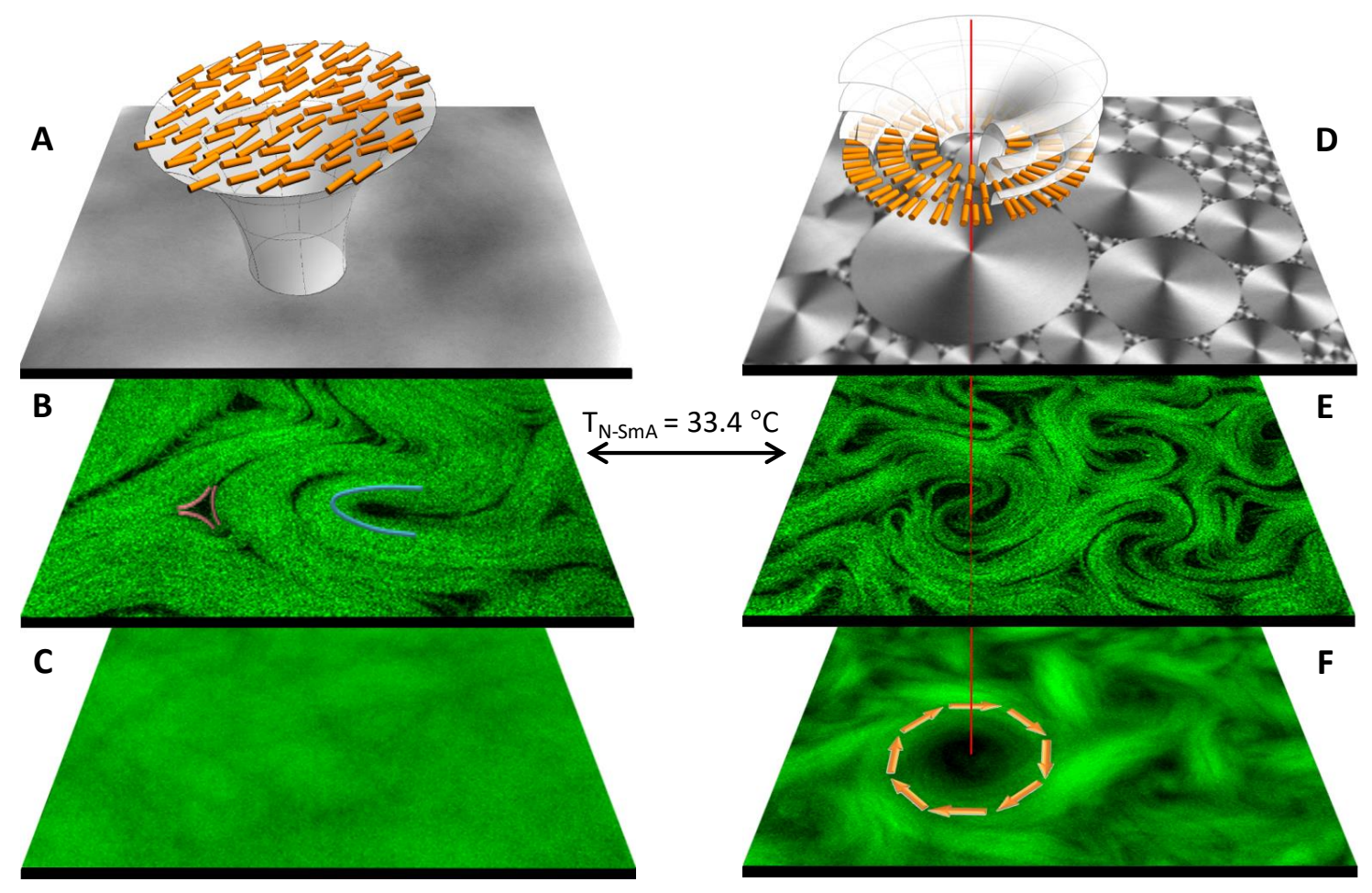

Figure 2. Self-assembly of the active layer in contact with the unforced liquid crystal. The active material is in contact either with the nematic (A-C) or the Smectic-A phase (D-F) of $8 \mathrm{CB}$. Reversible transition between both states is observed at $33.4^{\circ} \mathrm{C}$. Confocal reflection micrographs of the interface reveal the featureless nematic $8 \mathrm{CB}$ phase, where molecules lay, on average, parallel to the oil/water interface, with short-range positional but long-range orientational correlations (A). Fluorescence confocal microscopy imaging of the active nematic depleted towards the nematic $8 \mathrm{CB}$ (B) reveals the characteristic semi-integer folds (highlighted in B) that dynamically form, annihilate, and move in a turbulent fashion, with no temporal coherence, as evidenced by the time average shown in (C). A temperature quench into the lamellar Smectic-A phase of 8CB results in the formation of the well-known toroidal focal conic domains, as revealed by confocal reflection imaging (D). Fluorescence confocal microscopy, both with fast (E) and timeaveraged exposure $(\mathrm{F})$ reveal the formation of rotating mills in the active nematic, in registry with the circular domains in $8 \mathrm{CB}$. Micrographs are $244 \mu \mathrm{m}$ wide. 
(A)

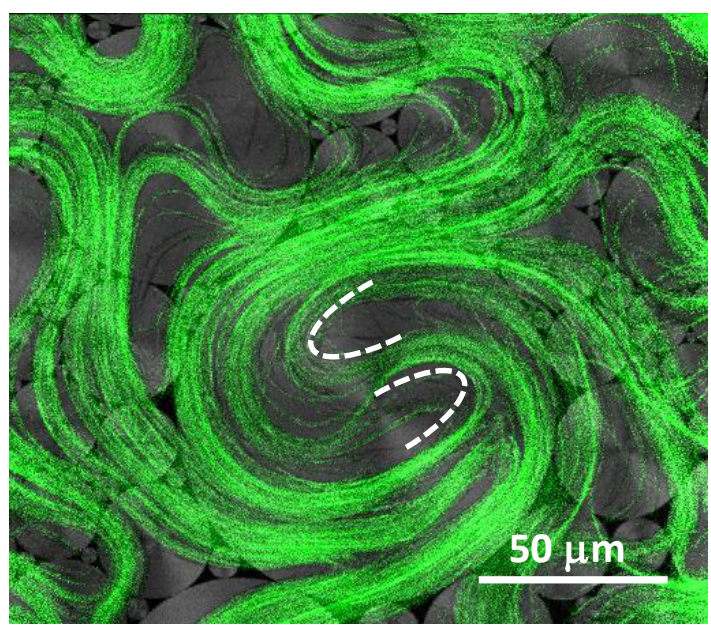

(B)

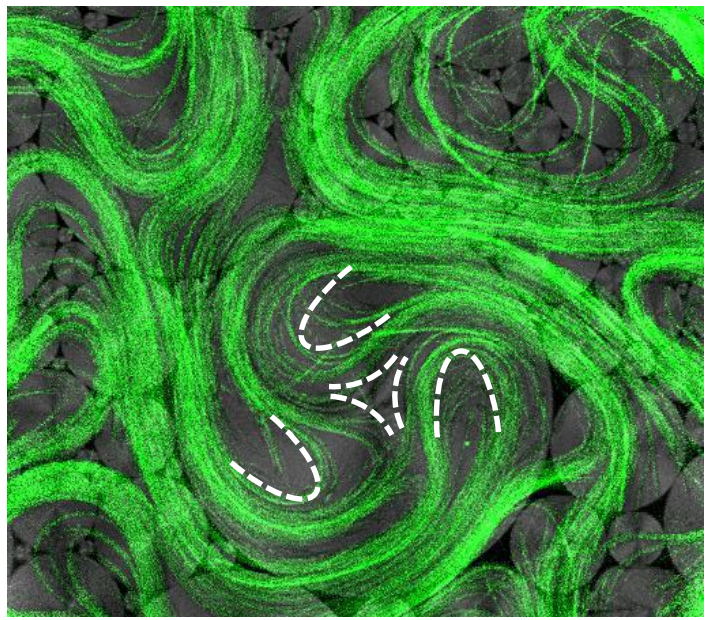

Figure 3. Fluorescence confocal micrograph showing two examples of the topological structure of a rotating mill of active nematic filaments preserves a constant topological charge $\mathrm{S}=+1$ at all times. Defects in the rotating mill are highlighted with dashed lines and have a charge (A) $2 \times 1 / 2$, and (B) $3 \times 1 / 2-1 / 2$.
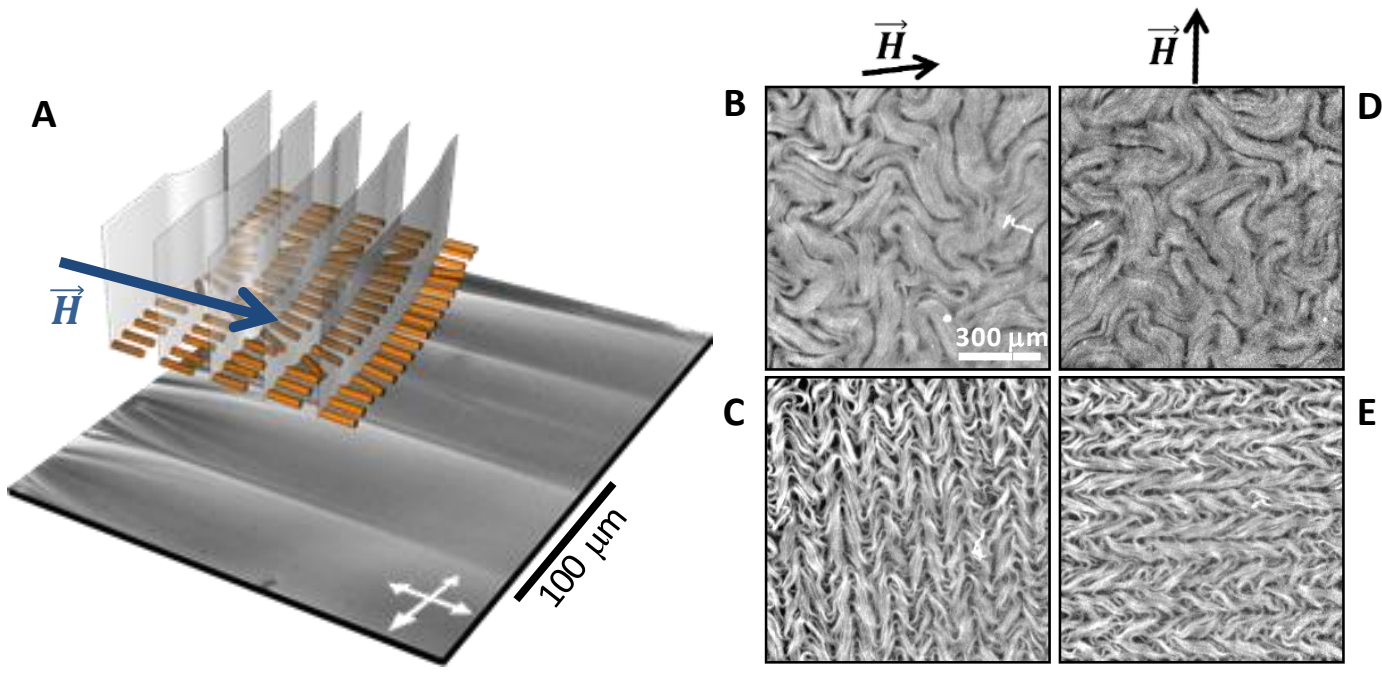

Figure 4. A: Polarized microscopy micrograph and sketch of the Smectic-A in the bookshelf configuration upon alignment with a magnetic field. B-D: Fluorescence micrographs of the active nematic interfaced with $8 \mathrm{CB}$ in different phases (Nematic in B, D; Smectic-A in C, E), in the presence of a magnetic field, as shown above each column. 
(A)

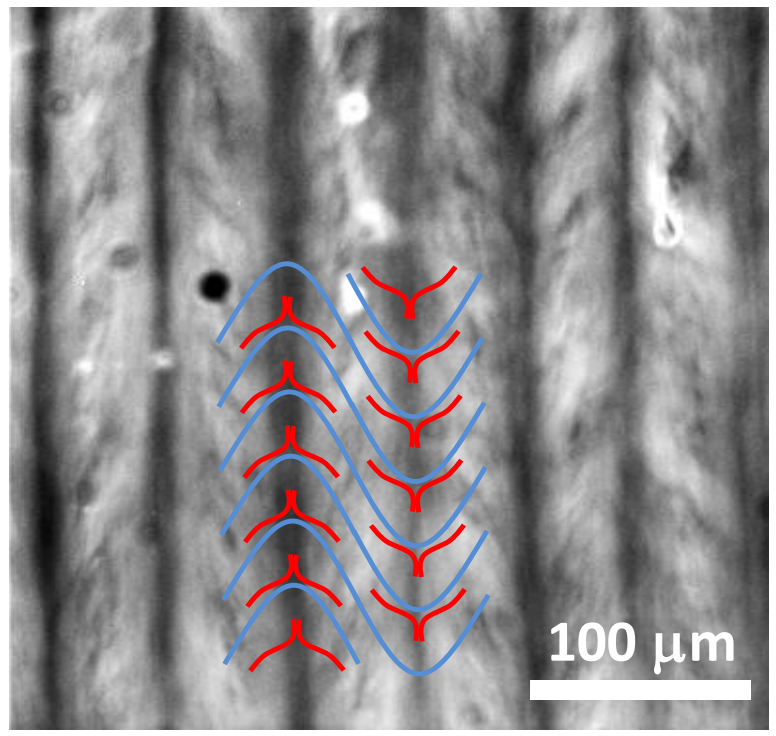

(B)

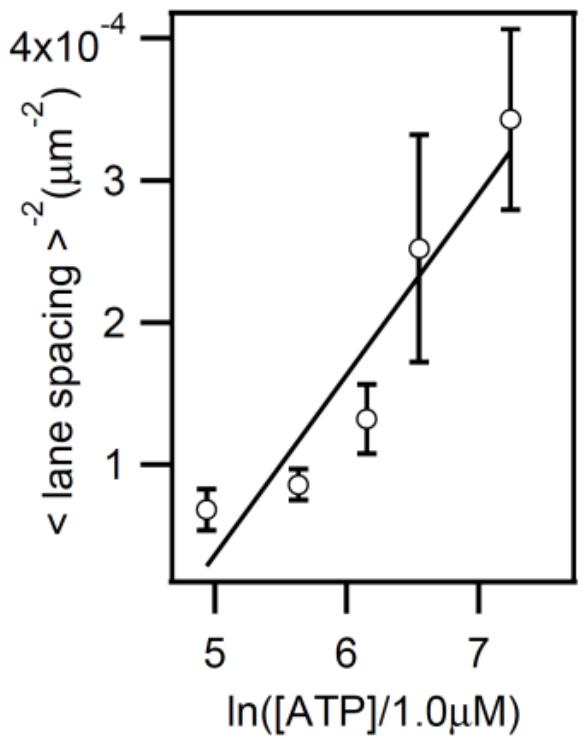

Figure 5. A: Fluorescence micrograph (long-time exposure) of the aligned active nematic. The lanes of alternated $+1 / 2$ (blue) and $-1 / 2$ (red) defects are shown. B: Dependence of lane spacing with activity. 

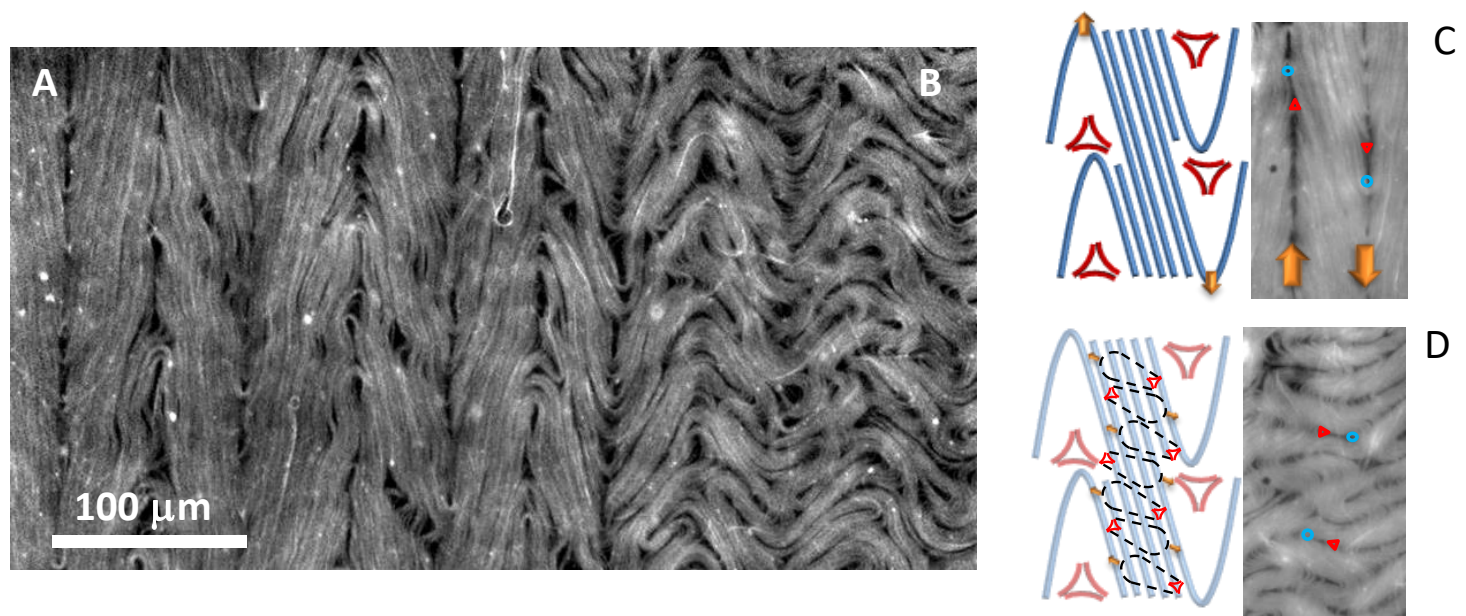

Figure 6. Fluorescence micrograph of the active nematic featuring the coexistence with a region with aligned stripes (A) and a region with the transversal instability (B). C, D: sketch of the configuration of the filaments, flow velocity, and defects in two adjacent flow lanes for the aligned and the unstable states, respectively. 
1. DeCamp, S. J.; Redner, G. S.; Baskaran, A.; Hagan, M. F.; Dogic, Z., Orientational order of motile defects in active nematics. Nat Mater 2015, 14, 1110-5.

2. Gao, T.; Blackwell, R.; Glaser, M. A.; Betterton, M. D.; Shelley, M. J., Multiscale polar theory of microtubule and motor-protein assemblies. Phys Rev Lett 2015, 114, 048101.

3. Karsenti, E.; Nedelec, F.; Surrey, T., Modelling microtubule patterns. Nat Cell Biol 2006, 8, 1204-11.

4. Kohler, S.; Schaller, V.; Bausch, A. R., Structure formation in active networks. Nat Mater 2011, 10, 462-8.

5. Nedelec, F. J.; Surrey, T.; Maggs, A. C.; Leibler, S., Self-organization of microtubules and motors. Nature 1997, 389, 305-8.

6. Sanchez, T.; Chen, D. T.; DeCamp, S. J.; Heymann, M.; Dogic, Z., Spontaneous motion in hierarchically assembled active matter. Nature 2012, 491, 431-4.

7. Schaller, V.; Bausch, A. R., Topological defects and density fluctuations in collectively moving systems. Proceedings of the National Academy of Sciences 2013, 110, 4488-4493.

8. Schaller, V.; Weber, C.; Semmrich, C.; Frey, E.; Bausch, A. R., Polar patterns of driven filaments. Nature 2010, 467, 73-7.

9. Schaller, V.; Weber, C. A.; Hammerich, B.; Frey, E.; Bausch, A. R., Frozen steady states in active systems. Proc Natl Acad Sci U S A 2011, 108, 19183-8.

10. Sumino, Y.; Nagai, K. H.; Shitaka, Y.; Tanaka, D.; Yoshikawa, K.; Chate, H.; Oiwa, K., Large-scale vortex lattice emerging from collectively moving microtubules. Nature 2012, 483, 448-52.

11. Surrey, T.; Nedelec, F.; Leibler, S.; Karsenti, E., Physical properties determining self-organization of motors and microtubules. Science 2001, 292, 1167-71.

12. Marchetti, M. C.; Joanny, J. F.; Ramaswamy, S.; Liverpool, T. B.; Prost, J.; Rao, M.; Simha, R. A., Hydrodynamics of soft active matter. Reviews of Modern Physics 2013, $85,1143-1189$.

13. Ramaswamy, S., The Mechanics and Statistics of Active Matter. Annual Review of Condensed Matter Physics 2010, 1, 323-345.

14. Oswald, P.; Pieranski, P., Smectic and columnar liquid crystals : concepts and physical properties illustrated by experiments. Taylor \& Francis: Boca Raton, FL, 2006; $\mathrm{p} \mathrm{xx}, 690 \mathrm{p}$.

15. Guillamat, P.; Ignes-Mullol, J.; Sagues, F., Control of active liquid crystals with a magnetic field. Proc Natl Acad Sci U S A 2016, 113, 5498-502. 
16. Henkin, G.; DeCamp, S. J.; Chen, D. T.; Sanchez, T.; Dogic, Z., Tunable dynamics of microtubule-based active isotropic gels. Philos Trans A Math Phys Eng Sci 2014, 372 .

17. Julicher, F.; Kruse, K.; Prost, J.; Joanny, J., Active behavior of the Cytoskeleton. Physics Reports 2007, 449, 3-28.

18. Kruse, K.; Joanny, J. F.; Julicher, F.; Prost, J.; Sekimoto, K., Generic theory of active polar gels: a paradigm for cytoskeletal dynamics. Eur Phys $J$ E Soft Matter 2005, $16,5-16$.

19. Prost, J.; Jülicher, F.; Joanny, J. F., Active gel physics. Nature Physics 2015, 11, 111-117.

20. Keber, F. C.; Loiseau, E.; Sanchez, T.; DeCamp, S. J.; Giomi, L.; Bowick, M. J.; Marchetti, M. C.; Dogic, Z.; Bausch, A. R., Topology and dynamics of active nematic vesicles. Science 2014, 345, 1135-9.

21. Giomi, L.; Bowick, M. J.; Ma, X.; Marchetti, M. C., Defect annihilation and proliferation in active nematics. Phys Rev Lett 2013, 110, 228101.

22. Shi, X. Q.; Ma, Y. Q., Topological structure dynamics revealing collective evolution in active nematics. Nat Commun 2013, 4, 3013.

23. Thampi, S. P.; Golestanian, R.; Yeomans, J. M., Velocity correlations in an active nematic. Phys Rev Lett 2013, 111, 118101.

24. Weber, C. A.; Bock, C.; Frey, E., Defect-mediated phase transitions in active soft matter. Phys Rev Lett 2014, 112, 168301.

25. Meyer, C.; Le Cunff, L.; Belloul, M.; Foyart, G., Focal Conic Stacking in Smectic A Liquid Crystals: Smectic Flower and Apollonius Tiling. Materials 2009, 2, 499-513.

26. Thampi, S. P.; Golestanian, R.; Yeomans, J. M., Vorticity, defects and correlations in active turbulence. Philos Trans A Math Phys Eng Sci 2014, 372.

27. Aditi Simha, R.; Ramaswamy, S., Hydrodynamic fluctuations and instabilities in ordered suspensions of self-propelled particles. Phys Rev Lett 2002, 89, 058101.

28. Voituriez, R.; Joanny, J. F.; Prost, J., Spontaneous flow transition in active polar gels. Europhysics Letters (EPL) 2005, 70, 404-410. 